\title{
Resonance Excitation of Slow Rotons: Linewidth
}

\author{
L. A. Melnikovsky \\ Institute for Physical Problems, Russian Academy of Sciences, ul. Kosygina 2, Moscow, 119334 Russia \\ e-mail: leva@kapitza.ras.ru
}

Received May 2, 2012; in final form, June 4, 2012

\begin{abstract}
An explanation of an anomalously narrow microwave absorption line in superfluid ${ }^{4} \mathrm{He}$ has been proposed. It has been shown that the experimentally observed resonance linewidth agrees with the assumption of parametric excitation of a macroscopic coherent roton state.
\end{abstract}

DOI: $10.1134 / \mathrm{S} 0021364012140081$

In their experimental investigation of the interaction of microwaves with superfluid ${ }^{4} \mathrm{He}$, Rybalko et al. [1] observed resonance absorption at the roton frequency $f_{0} \sim 180 \mathrm{GHz}$. Since the momentum of a photon is much lower than that of a roton, their transformation into one another is possible only near the wall, where only the energy is conserved. In this case, absorption would occur at an arbitrary frequency above the roton gap, $f>f_{0}$. However, the experimental absorption linewidth turned out to be anomalously narrow (the resolution of the spectrometer at low temperature is insufficient to measure the linewidth $\delta f \lesssim$ $50 \mathrm{kHz}$ ) and cannot be explained only by a singularity in the density of states of elementary excitations near the roton minimum.

To understand the nature of this absorption line, one has to take into account that rotons obey Bose statistics. A high population of a certain one-particle state stimulates further emission of rotons to the same state. Clearly, the population of the localized state is the greater the lower the velocity of particles escaping the active volume of the microwave resonator.

The linewidth can be quantitatively estimated within the above consideration. Rybalko et al. [1] used "whispering gallery" modes disk dielectric resonator with the radius $R \sim 1 \mathrm{~cm}$ immersed in liquid helium. The electric field in helium obeys the harmonic law $E(t)=E \cos 2 \pi f t$. The oscillation amplitude near the resonator wall is $E \sim 200 \mathrm{~V} / \mathrm{mm}$ and decays in the helium bulk at a characteristic distance of $L \sim 1 \mathrm{~mm}$. These oscillations (which modulate the energy spectrum of quasiparticles [2]) lead to the parametric creation of rotons.

The roton energy $\hbar \omega$ "oscillates" at the frequency $2 f$ :

$$
\hbar \omega(t) \sim \hbar \omega+\frac{(\epsilon-1)^{2} E^{2}(t) p_{0}^{2}}{8 \pi \hbar \rho \omega} \sim \hbar(\omega+\gamma \cos 4 \pi f t),
$$

where $\gamma=(\epsilon-1)^{2} E^{2} p_{0}^{2} /\left(16 \pi \hbar^{2} \rho \omega\right), \epsilon \sim 1.05$ is the dielectric constant of helium, $\rho=0.15 \mathrm{~g} / \mathrm{cm}^{3}$ is its mass density, and $p_{0}=2 \times 10^{-19} \mathrm{~cm} \mathrm{~g} / \mathrm{s}$ is the roton momentum. The parametric resonance occurs under the condition [3]

$$
|4 \pi f-2 \omega|<\sqrt{\gamma^{2}-4 \lambda^{2}} .
$$

The damping decrement $\lambda \sim v / L$ in Eq. (1) is determined by the escape of rotons with the velocity $v$ from the active volume of the resonator. The usual expression $\hbar \omega=2 \pi \hbar f_{0}+m v^{2} / 2$ for the energy of a slow roton, where $m \sim 0.16 m_{\mathrm{He}}$ is the effective mass, is substituted into Eq. (1). The maximization of the result with respect to $v$ gives

$$
f-f_{0}<\frac{\gamma^{2} L^{2} m}{16 \pi \hbar}+\frac{\hbar}{4 \pi m L^{2}} \approx 45 \mathrm{kHz} .
$$

The linewidth should increase with temperature: in addition to an increase in the natural uncertainty of energy, scattering of rotons impedes their escape from the active volume.

The resonance is due to the slowness of rotons. A similar effect could be observed in an arbitrary system in the vicinity of zero velocity of quasiparticles: maxons, magnons, photons in a metamaterial, etc.

I am grateful to A.F. Andreev and A.S. Rybalko for fruitful discussions. This work was supported by the Council of the President of the Russian Federation for Support of Young Scientists and Scientific Schools, project no. NSh-4889.2012.2.

\section{REFERENCES}

1. A. S. Rybalko, S. P. Rubets, E. Ya. Rudavskii, et al., Low Temp. Phys. 35, 837 (2009).

2. A. F. Andreev, JETP Lett. 31, 175 (1980).

3. L. D. Landau and E. M. Lifshitz, Course of Theoretical Physics, Vol. 1: Mechanics (Nauka, Moscow, 1982; Pergamon, New York, 1988), Sect. 27.

Translated by A. Safonov 